\title{
Relationship Between Coronary Contrast-Flow Quantitative Flow Ratio and Myocardial Ischemia Assessed by SPECT MPI
}

\author{
Jeff M. Smit ${ }^{1}$ - Gerhard Koning ${ }^{2}$ - Alexander R. van Rosendael ${ }^{1}$. \\ Petra Dibbets-Schneider ${ }^{3}$ - Bart J. Mertens ${ }^{4}$ - J. Wouter Jukema ${ }^{1}$ - Victoria Delgado ${ }^{1}$. \\ Johan H.C. Reiber ${ }^{2,5}$ • Jeroen J. Bax ${ }^{1}$ - Arthur J. Scholte ${ }^{1}$
}

Received: 16 May 2017 / Accepted: 26 June 2017 /Published online: 6 July 2017

(C) The Author(s) 2017. This article is an open access publication

\begin{abstract}
Purpose A new method has been developed to calculate fractional flow reserve (FFR) from invasive coronary angiography, the so-called "contrast-flow quantitative flow ratio (cQFR)". Recently, cQFR was compared to invasive FFR in intermediate coronary lesions showing an overall diagnostic accuracy of $85 \%$. The purpose of this study was to investigate the relationship between cQFR and myocardial ischemia assessed by single-photon emission computed tomography myocardial perfusion imaging (SPECT MPI).

Methods Patients who underwent SPECT MPI and coronary angiography within 3 months were included. The cQFR computation was performed offline, using dedicated software. The cQFR computation was based on 3-dimensional quantitative coronary angiography (QCA) and computational fluid dynamics. The standard 17-segment model was used to determine the vascular territories. Myocardial ischemia was defined as a summed difference score $\geq 2$ in a vascular territory. A cQFR of $\leq 0.80$ was considered abnormal.

Results Two hundred and twenty-four coronary arteries were analysed in 85 patients. Overall accuracy of cQFR to detect
\end{abstract}

\section{Arthur J. Scholte}

a.j.h.a.scholte@lumc.nl

1 Department of Cardiology, Leiden University Medical Center, Albinusdreef 2, Postal zone 2300 RC, Leiden, ZA 2333, The Netherlands

2 Medis medical imaging systems B.V., Leiden, The Netherlands

3 Department of Nuclear Medicine, Leiden University Medical Center, Leiden, The Netherlands

4 Department of Medical Statistics, Leiden University Medical Center, Leiden, The Netherlands

5 Department of Radiology, Leiden University Medical Center, Leiden, The Netherlands ischemia on SPECT MPI was 90\%. In multivariable analysis, cQFR was independently associated with ischemia on SPECT MPI (OR per 0.01 decrease of cQFR: 1.10; 95\% CI 1.04-1.18, $p=0.002$ ), whereas clinical and QCA parameters were not. Furthermore, cQFR showed incremental value for the detection of ischemia compared to clinical and QCA parameters (global chi square 48.7 to $62.6 ; p<0.001$ ).

Conclusions A good relationship between cQFR and SPECT MPI was found. cQFR was independently associated with ischemia on SPECT MPI and showed incremental value to detect ischemia compared to clinical and QCA parameters.

Keywords Computational fluid dynamics $\cdot$ Coronary artery disease $\cdot$ Fractional flow reserve $\cdot$ Non-invasive imaging . Quantitative coronary angiography

\begin{tabular}{ll}
\multicolumn{2}{l}{ Abbreviations } \\
AS & area stenosis \\
cQFR & contrast-flow quantitative flow ratio \\
DS & diameter stenosis \\
FFR & fractional flow reserve \\
LAD & left anterior descending coronary artery \\
LCX & left circumflex coronary artery \\
LL & lesion length \\
LVEF & left ventricular ejection fraction \\
MLD & minimal lumen diameter \\
PCI & percutaneous coronary intervention \\
QCA & quantitative coronary angiography \\
RCA & right coronary artery \\
SDS & summed difference score \\
SPECT & single-photon emission computed tomography \\
MPI & myocardial perfusion imaging
\end{tabular}




\section{Introduction}

Fractional flow reserve (FFR) is considered the gold standard to assess the hemodynamic significance of coronary artery stenoses [1]. In patients with multivessel coronary artery disease undergoing primary percutaneous coronary intervention (PCI), FFR-based revascularization is associated with improved clinical outcomes compared to angiography-based revascularization [2]. Therefore, according to recent guidelines of the European Society of Cardiology (ESC), FFR is recommended to identify hemodynamically relevant coronary artery stenosis when evidence of ischemia on non-invasive testing is not available [3]. Despite the supporting evidence for FFR assessment in intermediate coronary artery stenoses, FFR is used in only a minority of the cases (6.1\%) [4]. This may be related to some important limitations of FFR, such as the high costs of the pressure-wire and the need for hyperaemia induction.

To overcome these limitations, a new method has been developed to calculate FFR from invasive coronary angiography, the so-called "contrast-flow quantitative flow ratio (cQFR)" [5, 6]. This method enables a simple and fast computation of FFR based on 3-dimensional (3-D) quantitative coronary angiography (QCA) and computational fluid dynamics without the need of hyperaemia induction. Recently, cQFR was compared to invasive FFR in intermediate coronary lesions showing an overall diagnostic accuracy of detecting an invasive FFR $\leq 0.80$ of $85 \%$ with a sensitivity and specificity of $74 \%$ and $91 \%$, respectively [5].

cQFR has not yet been compared to non-invasive imaging methods for the identification of myocardial ischemia. Accordingly, the purpose of this study was to investigate (1) the relationship between cQFR and ischemia assessed by single-photon emission computed tomography myocardial perfusion imaging (SPECT MPI) and (2) the incremental value of $\mathrm{cQFR}$ compared to clinical and QCA parameters to detect ischemia.

\section{Materials and methods}

\section{Patients}

Patients who underwent SPECT MPI and coronary angiography within 3 months were included in the study. Patients with prior myocardial infarction, coronary artery bypass grafting, heart failure or uninterpretable SPECT MPI were excluded from the analysis. Clinical data were prospectively entered in the electronic patient file and retrospectively analysed. The Medical Ethical Committee of the Leiden University Medical Center, The Netherlands, approved this retrospective evaluation of clinically collected data and waived the need for written informed consent.

\section{cQFR analysis}

The cQFR analysis was performed offline, using a software package (QAngio XA 3D, Medis Medical Imaging Systems, Leiden, The Netherlands). Procedural details concerning cQFR analysis have been described previously [7]. In short, two angiographic views with projection angles at least $25^{\circ}$ apart comprising a minimum of vessel overlap and foreshortening were required for the analysis. The enddiastolic phase was selected in both angiographic views, after which the lumen and vessel wall contours were automatically detected and, if needed, manually adjusted. Subsequently, a 3$D$ reconstruction of the coronary artery was obtained and the QCA parameters for each coronary stenosis were readily available. To calculate the contrast-flow velocity, the contrast transport time in a specific coronary artery segment was determined using the Thrombolysis In Myocardial Infarction frame counting method [6]. The angiographic projection with the best contrast-flow image quality was used for this purpose. Finally, the contrast-flow velocity was utilized to calculate the hyperaemic flow velocity, from which the $\mathrm{CQFR}$ was derived.

The cQFR analysis was performed in epicardial coronary arteries and side branches with a quantitatively assessed diameter stenosis (DS) $\geq 1.4 \mathrm{~mm}$. The following QCA parameters were obtained: percentage DS, percentage area stenosis (AS), minimal lumen diameter (MLD) and lesion length (LL). Coronary arteries with chronic total occlusion, insufficient image quality or absence of angiographic views with projection angles at least $25^{\circ}$ apart were excluded from the analysis. For analysis purposes, coronary lesions in the diagonal and septal branches were allocated to the left anterior descending coronary artery (LAD) and lesions in the intermediate, anterolateral and obtuse marginal branches were allocated to the left circumflex coronary artery (LCX). If $>1$ stenosis was present in an epicardial coronary artery and/or the corresponding side branches, QCA parameters and cQFR were obtained for the stenosis and the coronary artery with the highest percentage DS, respectively. If no stenosis was present in an epicardial coronary artery (LAD, right coronary artery (RCA) or LCX) or any side branch: (1) QCA was performed for the mid-part of the epicardial coronary artery (LAD, RCA or LCX) as a reference and (2) cQFR was calculated for the epicardial coronary artery (LAD, RCA or LCX). A cQFR of $\leq 0.80$ was considered abnormal.

\section{SPECT MPI}

In all patients, gated SPECT MPI was performed within 3 months before coronary angiography. Patients were instructed to discontinue beta-blockers and calcium antagonists for at least 48 hours and caffeine use for at least 12 hours before the examination. In all patients, a two-day stress-rest protocol with technetium- $99 \mathrm{~m}\left({ }^{99 \mathrm{~m}} \mathrm{Tc}\right)$ tetrofosmin $(500 \mathrm{MBq})$ was 
performed. The stress method of first choice was bicycle ergometry, unless patients were unable to or had contraindications for physical exercise, in which case a pharmacological stress test with adenosine, dobutamine or dipyridamole was performed. During bicycle ergometry, tetrofosmin was injected at the moment of peak stress. Adenosine was administered in a dose of $140 \mu \mathrm{g} / \mathrm{kg} / \mathrm{min}$ for 6 minutes and tetrofosmin was injected 3 minutes after the start of the adenosine infusion. Dobutamine was administered for 15 minutes according to a predefined dosage scheme with an increasing dosage over time from 5 to $40 \mu \mathrm{g} / \mathrm{kg} / \mathrm{min}$. Subsequently, tetrofosmin was administered if the heart rate was at least $85 \%$ of the maximum heart rate. If the heart rate was still not adequate after dobutamine infusion, atropine sulfate was administered in a maximum dose of $1.0 \mathrm{mg}$. Dipyridamole was administered in a dose of $140 \mu \mathrm{g} / \mathrm{kg} / \mathrm{min}$ over a 4minute period, and tetrofosmin was injected.

SPECT MPI was performed 45 minutes after tetrofosmin administration with a triple-head (GCA 9300/HG; Toshiba Corporation, Tokyo, Japan) or dual-head SPECT camera system (GCA 7200/HG; Toshiba Corporation, Tokyo, Japan), both equipped with low-energy high-resolution collimators, using a 360 degrees acquisition mode. A $20 \%$ window was used around the $140 \mathrm{keV}$ energy peak of tetrofosmin, after which the SPECT data were stored in a 64 x 64 matrix. Stress and rest SPECT datasets were postprocessed using previously validated automated software [8]. Data were reconstructed in vertical and horizontal long-axes and short-axis views perpendicular to the heart axis. The ECG-gated SPECT data were used to determine the left ventricular ejection fraction (LVEF) at rest and during stress.

The 17-segment model was used to assign the myocardial segments to the three major coronary arteries $[9,10]$. In this model, the anteroseptal segments and apex belong to the LAD territory, the inferoseptal segments to the RCA territory and the lateral segments to the LCX territory. For every segment, a score was given from 0 to 4 based on tracer uptake $(0=$ normal tracer uptake; $1=$ mild reduction; $2=$ moderate reduction; $3=$ severe reduction; and $4=$ absent tracer uptake). Subsequently, the summed stress score (SSS) and summed rest score (SRS) were calculated for all patients by adding the scores of the different segments and the summed difference score (SDS) was calculated by subtraction of the SRS from the SSS. Myocardial ischemia was defined as a SDS $\geq 2$ in a vascular territory [11].

\section{Statistical analysis}

Distribution of continuous variables was evaluated using histograms and normal Q-Q plots. Normally distributed continuous variables are presented as mean \pm standard deviation and were compared using the independent sample Student-T test. Nonnormally distributed continuous variables are presented as median and $25-75 \%$ interquartile range (IQR) and were compared using the Mann-Whitney U test. Categorical variables are presented as number and percentages and compared with the chisquare test. A binomial logistic regression was performed to determine the effects of cQFR, QCA and clinical baseline parameters on the presence of myocardial ischemia in a vascular territory. The odds ratio (OR) and $95 \%$ confidence interval $(\mathrm{CI})$ were calculated for each variable in the analysis. Variables with a $\mathrm{p}$ value $<0.10$ in univariable analysis were entered in a multivariable model. To investigate the incremental value of cQFR to detect ischemia in a vascular territory over clinical and QCA parameters, three models were developed by introducing the following variables in a stepwise fashion: (1) clinical baseline parameters, (2) QCA parameters and (3) cQFR. Global chisquare values were calculated for each model and differences were compared using the likelihood ratio Chi-squared test. All statistical analyses were performed with the SPSS software package (IBM Corp. Released 2015. IBM SPSS Statistics for Windows, Version 23.0. Armonk, NY: IBM Corp.). A statistical test was considered significant if the $\mathrm{p}$-value was $<0.05$.

\section{Results}

\section{Patients}

In total, 85 patients who underwent SPECT MPI and coronary angiography within 3 months were included in the study. The median time between SPECT MPI and coronary angiography was 1.8 months (range 0.3-3.0). The clinical characteristics and SPECT MPI data of all study patients are shown in Tables 1 and 2 , respectively. Mean patient age was $66 \pm 11$ years, $66 \%$ were male, $59 \%$ had angina and $91 \%$ of patients had $\geq 1$ risk factor for cardiovascular disease. An adenosine stress test was performed in $72 \%$ of the patients before SPECT MPI, an exercise test in $22 \%$ and a dobutamine and dipyridamole stress test in $4 \%$ and $2 \%$ of the patients, respectively. LVEF was comparable at rest and during stress ( $67 \pm 9 \%$ vs. $66 \pm 10 \% ; p=0.58)$.

\section{Relationship between cQFR and SPECT MPI}

Of all patients, $31(36 \%)$ showed ischemia in $\geq 1$ vascular territory on SPECT MPI and median SDS of the overall population was 0 (IQR 0-4).

In total, 31 of 255 coronary arteries $(12 \%)$ were excluded from the cQFR analysis because of insufficient image quality $(n=8)$, coronary artery occlusion $(n=8)$, absence of angiographic projection angles $\geq 25^{\circ}$ apart $(n=6)$, overlap of coronary arteries $(n=6)$, distal location of coronary artery stenosis $(n=1)$, coronary artery spasm $(n=1)$ or bridging $(n=1)$.

In total, $37(16.5 \%)$ vascular territories showed ischemia on SPECT MPI and median SDS of the ischemic vascular territories was 3 (IQR 2-6). Moreover, 187 (83.5\%) vascular territories showed no ischemia on SPECT MPI and median SDS 
Table 1 Baseline characteristics

\begin{tabular}{ll}
\hline & Overall $(n=85)$ \\
\hline Age (years) & $66 \pm 11$ \\
Male, $n(\%)$ & $56(66 \%)$ \\
Hypertension, $n(\%)$ & $50(59 \%)$ \\
Hypercholesterolemia, $n(\%)$ & $43(51 \%)$ \\
Family history of CAD, $n(\%)$ & $35(41 \%)$ \\
Diabetes, $n(\%)$ & $33(39 \%)$ \\
Current smoker, $n(\%)$ & $20(24 \%)$ \\
Obesity $(\mathrm{BMI} \geq 30), n(\%)$ & $29(34 \%)$ \\
Symptoms, $n(\%)$ & \\
Chest pain & $50(59 \%)$ \\
Dyspnea & $23(27 \%)$ \\
Palpitations & $5(6 \%)$ \\
Fatigue & $23(27 \%)$ \\
Medication, $n(\%)$ & \\
Beta-blockers & $52(61 \%)$ \\
ACE-inhibitors/ARBs & $44(52 \%)$ \\
Statins & $59(69 \%)$ \\
Antiplatelet therapy & $52(61 \%)$ \\
Nitrates & $11(13 \%)$ \\
\hline AE &
\end{tabular}

$\mathrm{ACE}=$ angiotensin-converting enzyme; $\mathrm{ARB}=$ angiotensin-II-receptor blocker; $\mathrm{BMI}=$ body mass index; $\mathrm{CAD}=$ coronary artery disease

of the non-ischemic vascular territories was 0 (IQR $0-0)(p$ $<0.001$ compared to SDS of the ischemic vascular territories). In total, 16 (20.3\%) RCA vascular territories, 16 (21.3\%) LAD territories and five $(7.1 \%)$ LCX territories showed ischemia on SPECT MPI.

The cQFR was $\leq 0.80$ in 26 (11.6\%) coronary arteries and median cQFR was 0.96 (IQR 0.89-0.98) (Table 3). In coronary arteries with a cQFR $\leq 0.80,20$ (77\%) corresponding vascular territories showed ischemia on SPECT MPI (Fig. 1). In coronary arteries with a cQFR >0.80, 181 (91\%) corresponding vascular territories showed no ischemia on SPECT MPI. Also, SDS was significantly higher for coronary arteries with a cQFR $\leq 0.80$ compared to coronary arteries with a cQFR $>0.80$ (median SDS 3 (IQR 2-6) vs. 0 (IQR 0-0); $p<0.001$ ). Overall accuracy of cQFR to detect ischemia on SPECT MPI was 90\%. In Fig. 2, an example of a patient with an abnormal cQFR and ischemia on SPECT MPI is shown. In Fig. 3, an example of a patient with a normal cQFR and no ischemia on SPECT MPI is displayed.

\section{Incremental value of cQFR for detection of ischemia}

In multivariable analysis, each 0.01 decrease in CQFR was independently associated with ischemia on SPECT MPI (OR 1.10; $95 \%$ CI 1.04-1.18, $p=0.002$ ), whereas quantitatively assessed DS and LL were not (OR 1.01; 95\% CI 0.98-1.04 and OR 1.00; 95\% CI 0.93-1.07) (Table 4). Also, there was no association between any of the clinical baseline parameters and the presence
Table 2 SPECT MPI data

Overall $(n=85)$

$\begin{array}{ll}\text { Stress test, } n(\%) & \\ \text { Exercise } & 19(22 \%) \\ \text { Adenosine } & 61(72 \%) \\ \text { Dobutamine } & 3(4 \%) \\ \text { Dipyridamole } & 2(2 \%) \\ \text { Rest } & \\ \text { Heart rate (bpm) } & 79 \pm 18 \\ \text { Systolic blood pressure (mmHg) } & 146 \pm 22 \\ \text { Diastolic blood pressure (mmHg) } & 84 \pm 12 \\ \text { LVEF (\%) } & 67 \pm 9 \\ \text { Stress } & \\ \text { Heart rate (bpm) } & 116 \pm 31 \\ \text { Systolic blood pressure (mmHg) } & 172 \pm 34 \\ \text { Diastolic blood pressure (mmHg) } & 86 \pm 16 \\ \text { LVEF (\%) } & 66 \pm 10 \\ \text { Maximal exercise (Watt) } & 155 \pm 37 \\ \text { Validity (\%) } & 94 \pm 12 \\ \text { Symptoms during exercise, } n(\%) & 4(21 \%) \\ \text { ECG ischemia during exercise, } n(\%) & 6(32 \%) \\ \quad \text { SPECT MPI ischemia, } n(\%) & 2(33 \%) \\ \text { ECG no ischemia during exercise, } n(\%) & 13(68 \%) \\ \text { SPECT MPI no ischemia, } n(\%) & 9(69 \%)\end{array}$

Values are mean $\pm \mathrm{SD}$ or $n(\%)$.

$\mathrm{ECG}=$ electrocardiogram; $\mathrm{LVEF}=$ left ventricular ejection fraction; SPECT MPI = single-photon emission computed tomography myocardial perfusion imaging

of myocardial ischemia in a vascular territory. Addition of the QCA parameters to a model including clinical variables added significant incremental value (global chi-square 14.7 to $48.7 ; p$ $<0.001$ ) for the detection of ischemia (Fig. 4). Furthermore, addition of cQFR to the model containing the clinical and QCA variables added further incremental diagnostic value (global chisquare 48.7 to $62.6 ; p<0.001$ ).

\section{Discussion}

In this study, the relationship between cQFR and ischemia on SPECT MPI was investigated. In coronary arteries with an abnormal cQFR, 77\% of the corresponding vascular territories showed ischemia on SPECT MPI. Also, in coronary arteries with a normal cQFR, $91 \%$ of the corresponding vascular territories showed no ischemia on SPECT MPI. Overall accuracy of cQFR to detect ischemia on SPECT MPI was 90\%. cQFR was independently associated with ischemia on SPECT MPI, whereas clinical and QCA parameters were not. Finally, cQFR showed significant incremental value to detect ischemia compared to clinical and QCA parameters. 
Table 3 QCA parameters and $\mathrm{cQFR}$ for ischemic and nonischemic vascular territories

\begin{tabular}{lllll}
\hline & Overall $(n=224)$ & Ischemia $(\mathrm{n}=37)$ & No ischemia $(n=187)$ & P-value \\
\hline DS (\%) & $11.8(3.3-36.6)$ & $47.8(17.1-66.0)$ & $9.3(2.8-26.3)$ & $<0.001$ \\
LL (mm) & $0(0-8.7)$ & $13.2(0-20.4)$ & $0(0-4.4)$ & $<0.001$ \\
AS (\%) & $13.1(0-49.9)$ & $66.9(20.7-83.3)$ & $9.9(0-33.3)$ & $<0.001$ \\
MLD (mm) & $2.0 \pm 0.9$ & $1.6 \pm 0.9$ & $2.1 \pm 0.9$ & 0.001 \\
cQFR & $0.96(0.89-0.98)$ & $0.77(0.62-0.92)$ & $0.97(0.93-0.99)$ & $<0.001$ \\
\hline
\end{tabular}

Values are mean $\pm \mathrm{SD}$ or median (interquartile range).

$\mathrm{AS}=$ area stenosis; $\mathrm{cQFR}=$ contrast-flow quantitative flow ratio; $\mathrm{DS}=$ diameter stenosis; $\mathrm{LL}$ = lesion length; $\mathrm{MLD}=$ minimal lumen diameter; $\mathrm{QCA}=$ quantitative coronary angiography

\section{Relationship between QFR and invasive FFR}

For validation purposes, QFR has been compared to invasive FFR in prior studies [5, 6]. Recently, cQFR and invasive FFR were compared in 84 vessels in 73 patients with intermediate coronary artery stenoses [5]. In that study, the sensitivity, specificity, positive predictive value, negative predictive value and accuracy of cQFR to detect an invasive FFR $\leq 0.80$ were $74 \%$, $91 \%, 83 \%, 86 \%$ and $85 \%$, respectively. Also, a good correlation $(r=0.77 ; p<0.001)$ and agreement (mean difference 0.00 , standard deviation $0.06 ; p=0.90$ ) between cQFR and invasive FFR were noted. In another study, QFR was applied to angiographic projections recorded during hyperemia and compared with invasive FFR in 77 vessels in 68 patients with intermediate coronary artery stenoses [6]. The sensitivity, specificity, positive and negative predictive value, and
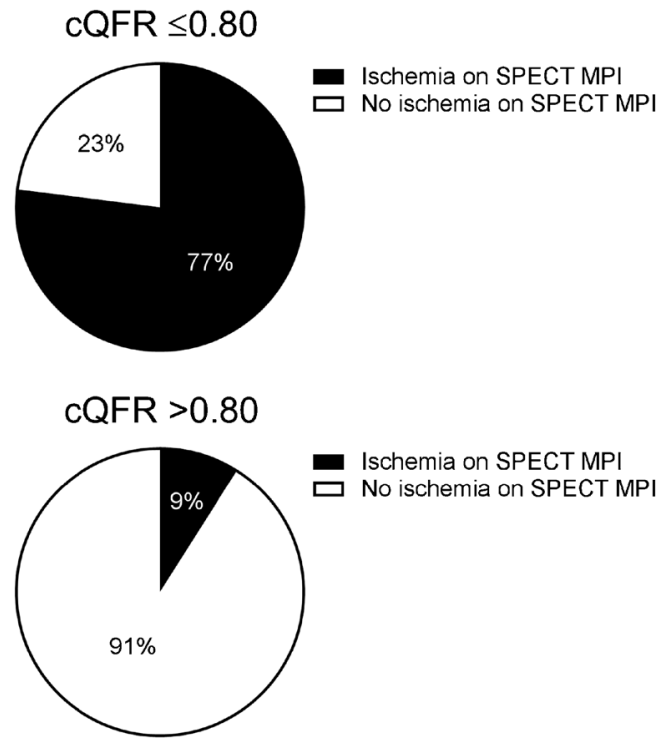

Fig. 1 Relationship between cQFR and SPECT MPI. In coronary arteries with a cQFR $\leq 0.80,77 \%$ of the corresponding vascular territories showed ischemia on SPECT MPI. Also, in coronary arteries with a cQFR $>0.80,91 \%$ of the corresponding vascular territories showed no ischemia on SPECT MPI. cQFR = contrast-flow quantitative flow ratio; SPECT MPI = single-photon emission computed tomography myocardial perfusion imaging accuracy of this so-called "adenosine-flow QFR (aQFR)" to detect an invasive FFR $\leq 0.80$ were $78 \%, 93 \%, 82 \%, 91 \%$ and $88 \%$, respectively. Also, a good correlation $(r=0.81 ; p$ $<0.001$ ) and agreement (mean difference 0.00 , standard deviation $0.06 ; p=0.54$ ) between aQFR and invasive FFR were observed.

\section{Relationship between cQFR and SPECT MPI}

The current study provides the first direct comparison between cQFR and SPECT MPI. We noted that $77 \%$ of the coronary arteries with a $\mathrm{cQFR} \leq 0.80$ showed ischemia on SPECT MPI. Furthermore, $91 \%$ of the coronary arteries with a cQFR $>0.80$ did not show ischemia on SPECT MPI. The results of the current study confirm the potential of cQFR to accurately detect ischemia on SPECT MPI, although these two modalities are based on different physiological concepts for the identification of hemodynamically significant coronary artery stenoses.

SPECT MPI determines the hemodynamic significance of coronary artery stenoses at the vascular territory level and is based on the physiological concept of relative flow reserve [12]. It compares the hyperaemic flow of a vascular territory supplied by a stenotic coronary artery to the hyperaemic flow of a vascular territory supplied by a non-stenotic coronary artery. As such, at least one normal vascular territory is needed for accurate detection of ischemia by SPECT MPI.

In contrast, the hemodynamic significance of coronary artery stenoses is determined at the coronary artery level by performance of $\mathrm{cQFR}[12,13]$. The $\mathrm{cQFR}$ can be precisely measured across the entire coronary artery or its side branches with high spatial resolution. Furthermore, cQFR is not dependent on the presence of coronary artery stenoses in adjacent vessels and is unique to each coronary artery or side branch.

The different physiological concepts of SPECT MPI and cQFR may result in discrepancy for assessment of ischemia. In patients with multivessel coronary artery disease, performance of SPECT MPI may lead to an underestimation of the hemodynamic significance of coronary artery lesions caused by "balanced ischemia". In a study by Melikian et al., 67 patients (201 vascular territories) with angiographically assessed 2- or 3-vessel coronary artery disease underwent SPECT MPI and coronary angiography 

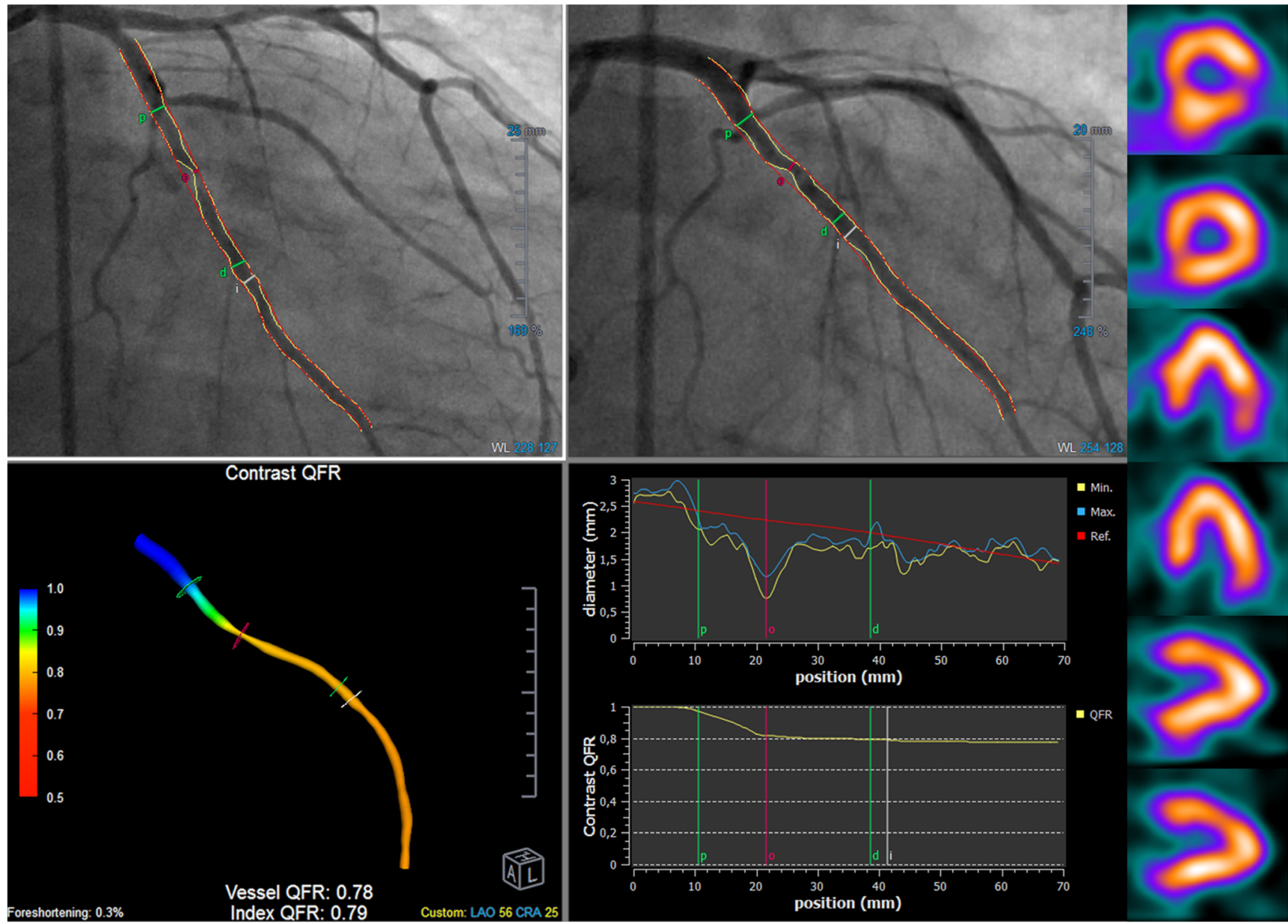

Fig. 2 Abnormal cQFR and ischemia on SPECT MPI. Example of a patient with a left circumflex coronary artery and a cQFR of 0.78 . SPECT MPI showed reversible perfusion defects in the left circumflex

vascular territory $(\operatorname{SDS}=6) . \mathrm{cQFR}=$ contrast-flow quantitative flow ratio; SDS = summed difference score; SPECT MPI = single-photon emission computed tomography myocardial perfusion imaging

with FFR assessment [12]. It was found that in $58 \%$ of patients with a discordant SPECT MPI and FFR, SPECT MPI underestimated the extent and severity of ischemia. Ragosta et al. analysed 88 coronary arteries in 36 patients with angiographically assessed 2- or 3-vessel coronary artery disease who underwent FFR and SPECT MPI [14]. Discordance between FFR and SPECT MPI was found in $31 \%$ of the vascular territories and was predominantly due to the absence of ischemia on SPECT MPI in vascular territories supplied by a coronary artery with a total occlusion or significant FFR value.

Discrepancy between SPECT MPI and cQFR may also be present in patients with coronary microvascular dysfunction caused by diabetes or hypertension [15]. This may lead to ischemia on SPECT MPI in the absence of epicardial coronary artery stenoses.

\section{Incremental value of cQFR for detection of ischemia}

In our study, cQFR was the only variable which was independently associated with ischemia on SPECT MPI. Furthermore,
cQFR showed incremental value for the detection of ischemia compared to clinical and QCA parameters.

The accuracy of QCA parameters for the assessment of ischemia is known to be limited. In a study by Yong et al., the relationship between QCA parameters and invasive FFR was investigated in 63 patients with intermediate coronary artery stenosis [16]. For percentage DS, the area under the receiver operating characteristic curve for detection of an FFR value $<0.80$ was 0.63 . A possible explanation for this finding could be that for FFR the maximum blood flow reduction is determined for the entire epicardial coronary artery, also when multiple stenoses are present, while for QCA the stenosis geometry is measured for only one lesion per coronary artery. Also, blood flow and mass of myocardium perfused by the coronary artery are not incorporated in the QCA analysis, although these are important factors for invasive FFR assessment. Our study showed that QCAbased computation of FFR, incorporating the concept of computational fluid dynamics and patient-specific flow, significantly improved the accuracy for the detection of 

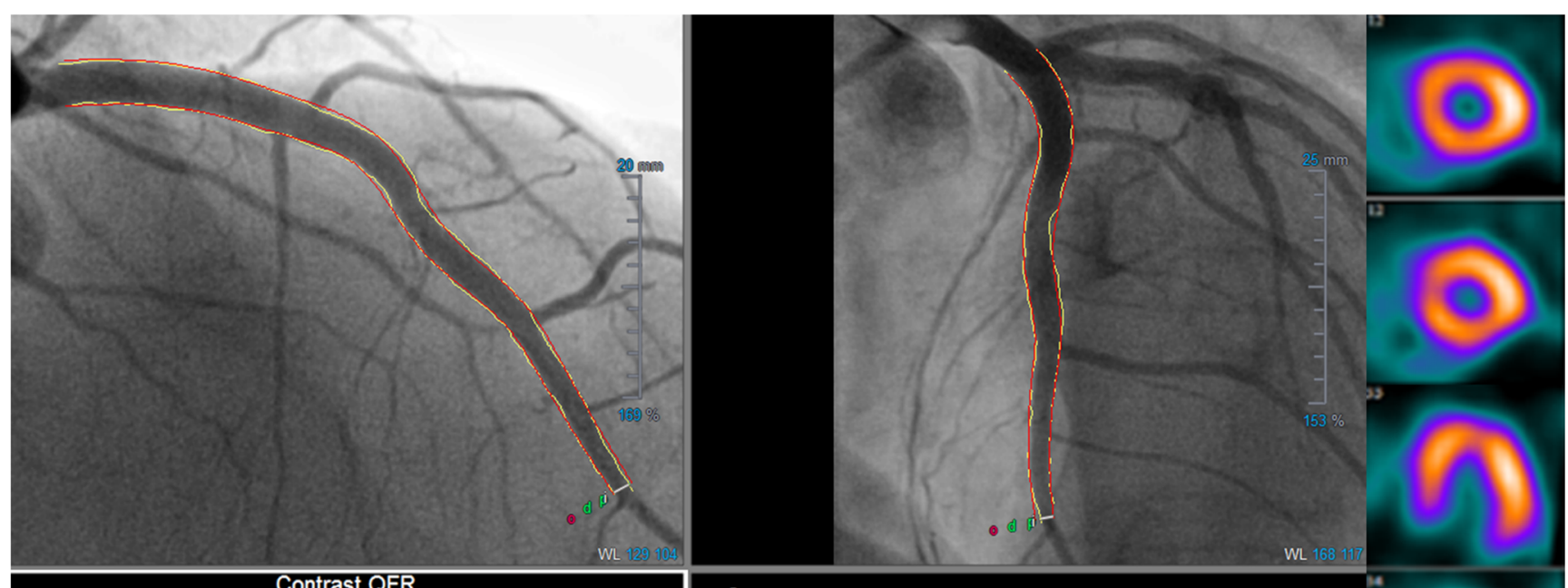

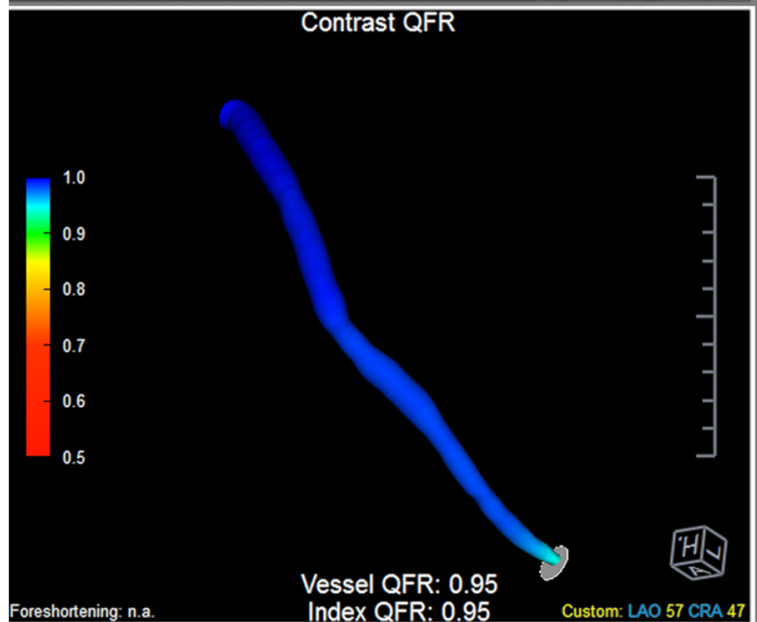

Fig. 3 Normal cQFR and no ischemia on SPECT MPI. Example of a patient with a left anterior descending coronary artery and a cQFR of 0.95. SPECT MPI showed no reversible perfusion defects. $\mathrm{cQFR}=$

ischemia on SPECT MPI. This finding supports the hypothesis that not only anatomical, but also functional

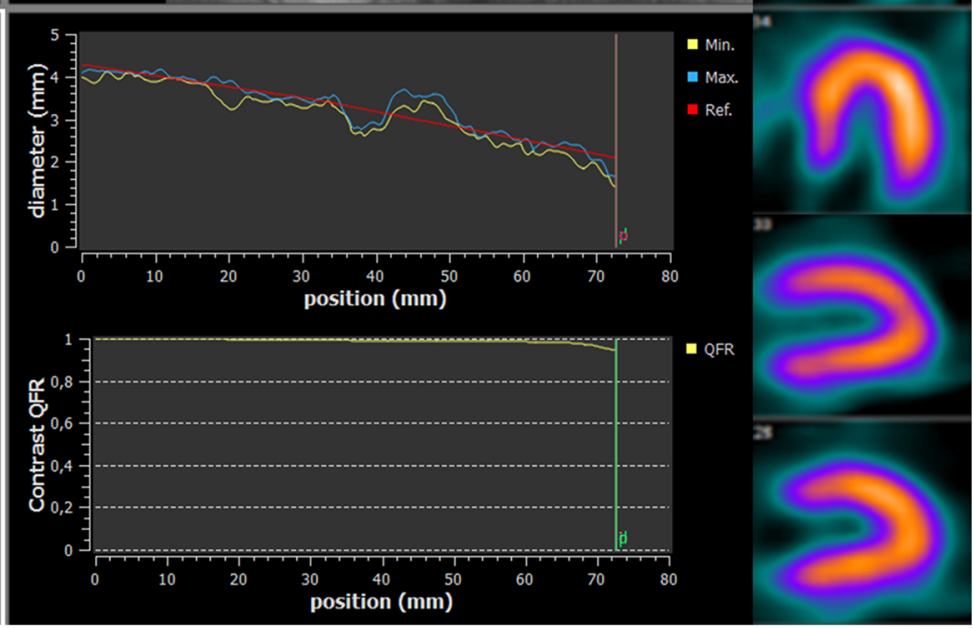

contrast-flow quantitative flow ratio; SPECT MPI = single-photon emission computed tomography myocardial perfusion imaging

parameters need to be incorporated for an accurate assessment of ischemia.
Table 4 Univariable and multivariable analysis for the detection of ischemia in a vascular territory

\begin{tabular}{|c|c|c|c|c|}
\hline & \multicolumn{2}{|l|}{ Univariable } & \multicolumn{2}{|l|}{ Multivariable } \\
\hline & OR $(95 \% \mathrm{CI})$ & P-value & OR $(95 \% \mathrm{CI})$ & P-value \\
\hline Age (per 1 year increase) & $1.06(1.02-1.10)$ & 0.005 & $1.03(0.98-1.08)$ & 0.23 \\
\hline Male & $1.06(0.50-2.21)$ & 0.89 & & \\
\hline Hypertension & $1.35(0.65-2.82)$ & 0.42 & & \\
\hline Hypercholesterolemia & $2.29(1.09-4.84)$ & 0.029 & $1.46(0.57-3.75)$ & 0.44 \\
\hline Family history of CAD & $1.16(0.57-2.37)$ & 0.68 & & \\
\hline Diabetes & $1.81(0.89-3.67)$ & 0.10 & $0.50(0.18-1.39)$ & 0.18 \\
\hline Current smoker & $1.08(0.47-2.46)$ & 0.86 & & \\
\hline Obesity (BMI $\geq 30$ ) & $0.71(0.32-1.56)$ & 0.40 & & \\
\hline DS (per 1\% increase) & $1.05(1.04-1.07)$ & $<0.001$ & $1.01(0.98-1.04)$ & 0.64 \\
\hline LL (per $1 \mathrm{~mm}$ increase) & $1.11(1.07-1.15)$ & $<0.001$ & $1.00(0.93-1.07)$ & 0.96 \\
\hline cQFR (per 0.01 decrease) & $1.12(1.08-1.16)$ & $<0.001$ & $1.10(1.04-1.18)$ & 0.002 \\
\hline
\end{tabular}

$\mathrm{BMI}=$ body mass index $\mathrm{CAD}=$ coronary artery disease $\mathrm{CI}=$ confidence interval; $\mathrm{cQFR}=$ contrast-flow quantitative flow ratio; $\mathrm{DS}=$ diameter stenosis; $\mathrm{LL}=$ lesion length; $\mathrm{OR}=$ odds ratio 


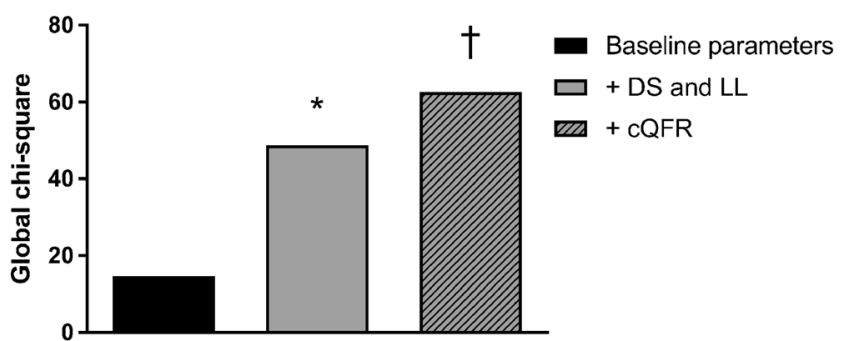

Fig. 4 Incremental value of cQFR compared to clinical and QCA parameters to detect ischemia in a vascular territory. Addition of the QCA parameters (diameter stenosis and lesion length) to a model including clinical variables added significant incremental value (* global chi-square 14.7 to $48.7 ; p<0.001$ compared to first model). Addition of cQFR to the model including clinical and QCA parameters added further incremental diagnostic value ( $\dagger$ global chi-square 48.7 to 62.6; $p<0.001$ compared to second model). cQFR = contrast-flow quantitative flow ratio; $\mathrm{DS}=$ diameter stenosis; $\mathrm{LL}=$ lesion length; $\mathrm{QCA}=$ quantitative coronary angiography

\section{Limitations}

This study is a retrospective study with all its inherent limitations. Selection bias may be introduced, because no standardized criteria were determined for performance of SPECT MPI and coronary angiography. Patients without ischemia on SPECT MPI who did not undergo coronary angiography were not included in the analysis. These patients could potentially have had "balanced ischemia" and significant cQFR values for all three epicardial coronary arteries, resulting in increased discrepancy between SPECT MPI and cQFR. Also, the use of the 17-segment model for the assignment of the myocardial segments to the three major coronary arteries is not ideal, because of anatomical variability.

\section{Conclusions}

A good relationship between cQFR and ischemia on SPECT MPI was found. cQFR was independently associated with ischemia on SPECT MPI. Furthermore, cQFR showed incremental value to detect ischemia compared to clinical and QCA parameters. Currently, cQFR offers additional value for the detection of hemodynamically significant lesions beyond clinical and QCA parameters, when invasive FFR is not available. Before $\mathrm{cQFR}$ can be adopted online at the catheterization laboratory as a potential alternative for invasive FFR, larger validation and outcome studies are needed.

\section{Compliance with ethical standards}

Disclosure of potential conflicts of interest The department of cardiology received research grants from Biotronik, Medtronic, Boston Scientific and Edwards Lifesciences. Arthur J. Scholte received consulting fees from Toshiba Medical Systems and GE Healthcare. Victoria Delgado received speaker fees from Abbott Vascular. Johan H.C. Reiber is the CEO of Medis and has a part time appointment at LUMC as professor of medical imaging. Gerhard Koning is an employee of Medis.

Ethical approval All procedures performed in studies involving human participants were in accordance with the ethical standards of the institutional and/or national research committee and with the 1964 Helsinki Declaration and its later amendments or comparable ethical standards.

Informed consent For this type of study formal consent is not required.

Open Access This article is distributed under the terms of the Creative Commons Attribution 4.0 International License (http:// creativecommons.org/licenses/by/4.0/), which permits unrestricted use, distribution, and reproduction in any medium, provided you give appropriate credit to the original author(s) and the source, provide a link to the Creative Commons license, and indicate if changes were made.

\section{References}

1. Pijls NH, Tanaka N, Fearon WF. Functional assessment of coronary stenoses: can we live without it? Eur Heart J. 2013;34(18):1335-44.

2. Pijls NH, Fearon WF, Tonino PA, Siebert U, Ikeno F, Bornschein B, et al. Fractional flow reserve versus angiography for guiding percutaneous coronary intervention in patients with multivessel coronary artery disease: 2-year follow-up of the FAME (Fractional Flow Reserve Versus Angiography for Multivessel Evaluation) study. J Am Coll Cardiol. 2010;56(3):177-84.

3. Montalescot G, Sechtem U, Achenbach S, Andreotti F, Arden C, Budaj A, et al. 2013 ESC guidelines on the management of stable coronary artery disease: the Task Force on the management of stable coronary artery disease of the European Society of Cardiology. Eur Heart J. 2013;34(38):2949-3003.

4. Dattilo PB, Prasad A, Honeycutt E, Wang TY, Messenger JC. Contemporary patterns of fractional flow reserve and intravascular ultrasound use among patients undergoing percutaneous coronary intervention in the United States: insights from the National Cardiovascular Data Registry. J Am Coll Cardiol. 2012;60(22): 2337-9.

5. Tu S, Westra J, Yang J, von Birgelen C, Ferrara A, Pellicano M, et al. Diagnostic Accuracy of Fast Computational Approaches to Derive Fractional Flow Reserve From Diagnostic Coronary Angiography: The International Multicenter FAVOR Pilot Study. JACC Cardiovasc Interv. 2016;9(19):2024-35.

6. Tu S, Barbato E, Koszegi Z, Yang J, Sun Z, Holm NR, et al. Fractional flow reserve calculation from 3-dimensional quantitative coronary angiography and TIMI frame count: a fast computer model to quantify the functional significance of moderately obstructed coronary arteries. JACC Cardiovasc Interv. 2014;7(7):768-77.

7. Tu S, Xu L, Ligthart J, Xu B, Witberg K, Sun Z, et al. In vivo comparison of arterial lumen dimensions assessed by coregistered three-dimensional (3D) quantitative coronary angiography, intravascular ultrasound and optical coherence tomography. Int J Cardiovasc Imaging. 2012;28(6):1315-27.

8. Germano G, Kavanagh PB, Waechter P, Areeda J, Van Kriekinge S, Sharir T, et al. A new algorithm for the quantitation of myocardial perfusion SPECT. I: technical principles and reproducibility. J Nucl Med. 2000;41(4):712-9.

9. Imaging guidelines for nuclear cardiology procedures, part 2. American Society of Nuclear Cardiology. J Nucl Cardiol. 1999;6(2):G47-84.

10. Cerqueira MD, Weissman NJ, Dilsizian V, Jacobs AK, Kaul S, Laskey WK, et al. Standardized myocardial segmentation and 
nomenclature for tomographic imaging of the heart. A statement for healthcare professionals from the Cardiac Imaging Committee of the Council on Clinical Cardiology of the American Heart Association. Circulation. 2002;105(4):539-42.

11. Sharir T, Germano G, Kang X, Lewin HC, Miranda R, Cohen I, et al. Prediction of myocardial infarction versus cardiac death by gated myocardial perfusion SPECT: risk stratification by the amount of stress-induced ischemia and the poststress ejection fraction. J Nucl Med. 2001;42(6):831-7.

12. Melikian N, De Bondt P, Tonino P, De Winter O, Wyffels E, Bartunek J, et al. Fractional flow reserve and myocardial perfusion imaging in patients with angiographic multivessel coronary artery disease. JACC Cardiovasc Interv. 2010;3(3): 307-14.
13. De Bruyne B, Sarma J. Fractional flow reserve: a review: invasive imaging. Heart. 2008;94(7):949-59.

14. Ragosta M, Bishop AH, Lipson LC, Watson DD, Gimple LW, Sarembock IJ, et al. Comparison between angiography and fractional flow reserve versus single-photon emission computed tomographic myocardial perfusion imaging for determining lesion significance in patients with multivessel coronary disease. Am J Cardiol. 2007;99(7):896-902.

15. Camici PG, Crea F. Coronary microvascular dysfunction. N Engl J Med. 2007;356(8):830-40

16. Yong $\mathrm{AS}, \mathrm{Ng} \mathrm{AC}$, Brieger D, Lowe $\mathrm{HC}, \mathrm{Ng}$ MK, Kritharides L. Three-dimensional and two-dimensional quantitative coronary angiography, and their prediction of reduced fractional flow reserve. Eur Heart J. 2011;32(3):345-53. 